\title{
Association between musculoskeletal function deterioration and locomotive syndrome in the general elderly population: a Japanese cohort survey randomly sampled from a basic resident registry
}

Ryosuke Tokida ${ }^{1}$, Shota Ikegami ${ }^{1,2^{*}}$ (D), Jun Takahashi ${ }^{2}$, Yoshikazu Ido $^{1}$, Ayaka Sato ${ }^{1}$, Noriko Sakai ${ }^{3}$, Hiroshi Horiuchi ${ }^{1}$ and Hiroyuki Kato ${ }^{1,2}$

\begin{abstract}
Background: Locomotive syndrome (LOS) is defined as the loss of mobility due to age-related impairment of motor organs. The purpose of this study was to evaluate the prevalence and severity of LoS, muscular strength and balancing ability, and prevalence of sarcopenia in relation to the presence of LoS according to sex and age groups ranging between 50 and 89 years.

Methods: Male and female participants between the ages of 50-89 were randomly selected in the resident registry of a cooperating town. Calls for participation continued until approximately 50 consenting participants were successfully recruited for each age group and sex. A total of 413 participants (203 male and 210 female) were enrolled for undergoing a LoS risk test and measuring their physical function. Physical function was compared to participants with or without LoS.

Results: A total of 312 patients (75.5\%) were diagnosed as LoS, of which 144 (46.2\%) were male and $168(53.8 \%)$ were female. The severity of LoS for the 312 patients were 210 (67.3\%) for stage 1 and 102 (32.7\%) for stage 2. The prevalence of LoS in males were 37,59,91, and 100\% in the 50s, 60s, 70s, and 80s age strata, respectively. The prevalence of LoS in females were 71,62,89, and 98\% in the 50s, 60s, 70s, and 80s age strata, respectively. The prevalence of sarcopenia was significantly higher as the age strata in males grew higher. Knee extension strength was significantly lower for participants in their 50 s and females in addition to females in their 60s with LoS. The 31 patients diagnosed as sarcopenia included 29 (93.5\%) with LoS, 11 (35.4\%) classified as LoS stage 1, and 18 (58.1\%) (Continued on next page)
\end{abstract}

\footnotetext{
* Correspondence: sh.ikegami@gmail.com

'Rehabilitation Center, Shinshu University Hospital, Asahi 3-1-1, Matsumoto, Nagano 390-8621, Japan

${ }^{2}$ Department of Orthopaedic Surgery, Shinshu University School of Medicine, Asahi 3-1-1, Matsumoto, Nagano 390-8621, Japan

Full list of author information is available at the end of the article
}

C C The Author(s). 2020 Open Access This article is licensed under a Creative Commons Attribution 4.0 International License, which permits use, sharing, adaptation, distribution and reproduction in any medium or format, as long as you give appropriate credit to the original author(s) and the source, provide a link to the Creative Commons licence, and indicate if changes were made. The images or other third party material in this article are included in the article's Creative Commons licence, unless indicated otherwise in a credit line to the material. If material is not included in the article's Creative Commons licence and your intended use is not permitted by statutory regulation or exceeds the permitted use, you will need to obtain permission directly from the copyright holder. To view a copy of this licence, visit http://creativecommons.org/licenses/by/4.0/. The Creative Commons Public Domain Dedication waiver (http://creativecommons.org/publicdomain/zero/1.0/) applies to the data made available in this article, unless otherwise stated in a credit line to the data. 
(Continued from previous page)

classified as stage 2.

Conclusions: The prevalence of LoS was high in participants over 70 years of age. In males, the prevalence of sarcopenia was higher as the age strata grew higher. Patients with LoS exhibited lower knee extension strength. We believe that some measures to prevent or improve LoS may require exercise to increase the muscle strength of the lower limbs.

Keywords: Epidemiological study, Cross-sectional survey, Prevalence, Sarcopenia, Locomotion, physical examination

\section{Background}

Japan is one of the most aged countries in the world, and the growth of its elderly population that require nursing care has become a significant social and financial problem. In 2007, the Japanese Orthopaedic Association proposed the term "locomotive syndrome" (LoS) to describe the loss of mobility due to age-related impairment of motor organs, and the organization subsequently launched an early detection and preventative care program $[1,2]$. Preventative care for LoS is important, as there is an increased risk of requiring nursing care with the progression of LoS.

In the past, only subjective evaluations such as a "lococheck" (a neologism combining "locomotive syndrome" and "check") and the 25-question geriatric locomotive function scale (25-qGLFS) [3] were conventionally used as a method for detecting LoS. However, the LoS risk test was developed in 2013 to enable objective evaluations, comprising of three risk assessments that include the stand-up test (SUT), two-step test (TST), and 25qGLFS. The severity of LoS can be classified into two stages under the LoS risk test, wherein LoS Stage 1 represents a mild stage that exhibits the initial loss of mobility and LoS Stage 2 represents a severe stage that exhibits a progressive loss of mobility [4]. Although surveys on the prevalence and severity of LoS in middleaged and elderly patients using the LoS risk test have been reported in the literature [5-8], to the best of our knowledge, only one report conducted examinations according to each age group and sex [5]. In addition, despite reports that suggest the muscle strength of the lower limbs, balancing ability, and walking ability were significantly related to LoS [9-11], few studies have examined the muscular strength and balancing ability in relation to the presence of $\operatorname{LoS}$ according to each age group and sex [5]. The first aim of this study was to clarify the association between physical function and LoS.

Sarcopenia is a condition characterized by muscle weakness, decline of motor ability, or loss of muscle mass due to aging [12]. In addition, sarcopenia is reported to be common in patients requiring long-term care and patients with motor disorders [13]. The clinical features of LoS and sarcopenia were closely linked to each other [14]. However, reports on the relationship between LoS and sarcopenia are scarce in the literature [15]. The second aim of this study was to clarify the association between LoS and sarcopenia.

Taking account of the background described above, we performed a cross-sectional study on the musculoskeletal function of a town resident. We evaluated the prevalence and severity of LoS according to sex and age groups ranging from 50 to 89 years, as well as the prevalence of sarcopenia. Furthermore, we assessed the knee extension strength, balancing ability, and sarcopenia in relation to LoS.

\section{Methods \\ Subjects}

This was cross-sectional study for observational research. From October 2014 to June 2017, we conducted an epidemiological study of residents (the Obuse Study) as a joint collaboration with a cooperating town office. Male and female participants between the ages of 50-89 were randomly selected from a pool of 5352 registrants in the resident registry of the cooperating town, which locates in a rural area. Those selected from the registry were asked whether they would be able to undergo a physical examination, and calls for participation were continued until approximately 50 consenting participants were successfully recruited for each age group, which were 50's, 60's, 70's, and 80's, and sex. Four hundred and fifteen participants were enrolled in the Obuse study. Four hundred and thirteen participants were consequently included in the study, excluding 2 participants with incomplete measurements (Fig. 1). Prior to this study, we had not performed a calculation to establish a statistical sample size based on multiple outcome indices. A feasible survey was conducted based on the balance between financial costs and time, number of staff, and participants.

\section{Assessing the presence / absence of LoS and its severity}

Prior to the physical examination, we mailed and requested a 25-qGLFS questionnaire [3] to be filled out by our participants. These self-reported questionnaires were collected on-site, where the examination was held. The SUT [16] and TST [17] were conducted during the examination. The SUT evaluates lower-body muscle 
strength by measuring whether the subject can rise from a sitting position on boxes of heights of $40,30,20$, and $10 \mathrm{~cm}$ with 1 or both legs. The relative difficulty of standing from each height, from least to most challenging, was as follows: $40 \mathrm{~cm}$, both legs standing (level 1); $30 \mathrm{~cm}$, both legs standing (level 2); $20 \mathrm{~cm}$, both legs standing (level 3); $10 \mathrm{~cm}$, both legs standing (level 4); 40 $\mathrm{cm}$, single-leg standing (level 5); $30 \mathrm{~cm}$, single-leg standing (level 6); $20 \mathrm{~cm}$, single-leg standing (level 7); and 10 $\mathrm{cm}$, single-leg standing (level 8). The successfully completed task with the greatest difficulty level was regarded as the subject's final grade. The 2-step test evaluated the total length of 2 maximally long strides (in centimetres) divided by body height (in centimetres).

LoS were established in two stages. From these three tests, the participants were classified into no LoS, LoS Stage 1, or LoS Stage 2 group. LoS stage 1 was defined as a TST score $<1.3$, difficulty with one-leg standing (OLS) from a $40 \mathrm{~cm}$ seat in the SUT (either leg), or a 25 -qGLFS score $\geq 7$. LoS stage 2 was defined as a TST score $<1.1$, difficulty with standing from a $20 \mathrm{~cm}$ seat using both legs in the SUT, or a 25 -qGLFS score $\geq 16$. LoS stage 1 was diagnosed as starting to decline in mobility, and LoS stage 2 was diagnosed as progressing to a decline in mobility [4].

\section{Evaluation of physical function}

The muscle strength for the lower limb was measured by a performance recorder (HUR ${ }^{\circ}$, Kokkola, Finland) and leg extension/curl Rehab 5530 (HUR •, Kokkola, Finland). The validity and reliability of these instruments have been reported [18] and have been commonly used in previous assessments of muscle strength $[19,20]$. The

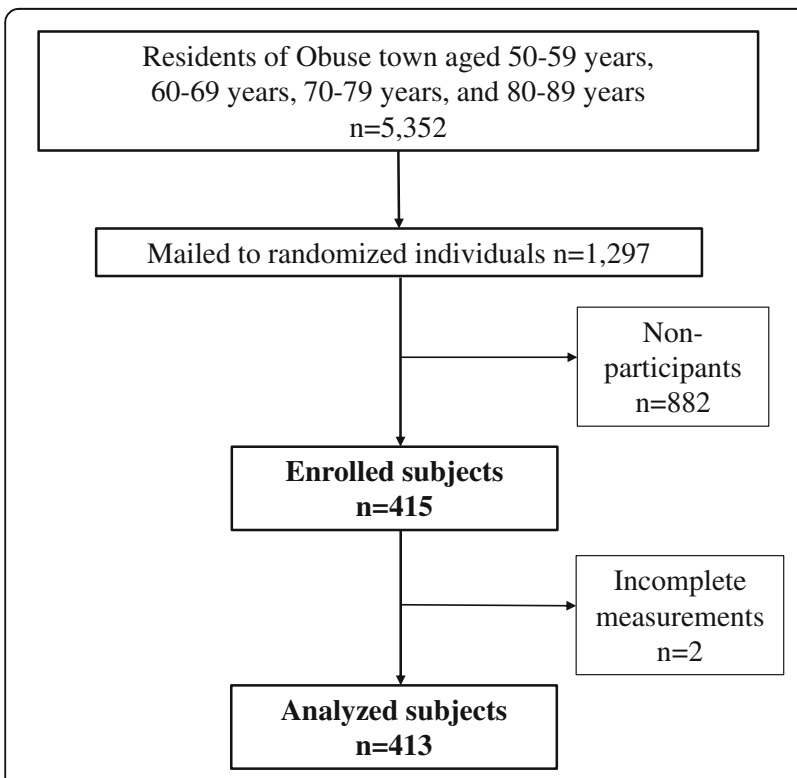

Fig. 1 Flow-chart of participants from a cooperating town right and left leg extension power $(\mathrm{kg})$ was divided by weight $(\mathrm{kg})$, and their mean value was defined as the knee extension strength. Balancing ability was measured by measuring the total duration the subject could stay in a balancing position while keeping his or her eyes open and standing on either leg [21]. At a maximum of $60 \mathrm{~s}$, the left and right mean values were defined as the OLS test (sec). The grip strength was measured using a grip strength meter (Jamar Hand Dynamometer, Sammons Preston Rolyan ${ }^{\circledR}$, Bolingbrook, Ill, USA), and the mean value on the left and right were defined as the grip strength.

\section{Assessment of sarcopenia}

We assessed the muscle mass and strength based on the criteria proposed by the Asian Working Group for Sarcopenia [12]. For determining the muscle mass, we used a Dual-Energy X-Ray Absorptiometry (GE Prodigy, GE healthcare, Chicago, IL, USA) to measure and evaluate the skeletal muscle mass index (SMI). In case the walking speed was less than $0.8 \mathrm{~m} / \mathrm{s}$ or the grip strength was less than $26 \mathrm{~kg}$ in males and less than $18 \mathrm{~kg}$ in females, the muscle mass was measured. The cut-off for muscle mass was $7.0 \mathrm{~kg} / \mathrm{m}^{2}$ in males and $5.4 \mathrm{~kg} / \mathrm{m}^{2}$ in females. Sarcopenia can be diagnosed based on having a low muscle mass that falls below the cut-off value. In the present study, we could not measure the walking speed due to timewise and spatial restrictions, so we used the grip strength as a primary physical performance assessment.

\section{Statistical analysis}

The prevalence of LoS for each age group and sex was compared to the prevalence of sarcopenia using the Steel-Dwass test. Knee extension strength and OLS test were compared to participants with or without LoS using the Student's t-test. The relationship between LoS and sarcopenia was analysed using the Fisher's exact test. Sensitivity, specificity, and positive / negative likelihood ratio of LoS diagnosis by sarcopenia were calculated. A positive likelihood ratio of more than 5 and a negative likelihood ratio of less than 0.2 were considered useful for diagnosis. All statistical analyses were performed with EZR (Saitama Medical Center, Jichi Medical University) [22], which is a graphical user interface for $R$ (version 3.5.2; The R Foundation for Statistical Computing, Vienna, Austria). $P<0.05$ was considered to be statistically significant.

\section{Results}

The baseline characteristics and functions of 413 participants are shown for each age group and sex in Table 1 . The number of male participants were 50 for those aged $50-59$ years, 53 for $60-69$ years, 55 for $70-79$ years, and 
Table 1 Characteristics of 413 subjects in the Obuse study cohort

\begin{tabular}{llllllll}
\hline & $\begin{array}{l}\text { Age strata } \\
\text { (years) }\end{array}$ & $\mathrm{N}$ & $\begin{array}{l}\text { Height } \\
(\mathrm{cm})\end{array}$ & $\begin{array}{l}\text { Weight } \\
(\mathrm{kg})\end{array}$ & $\begin{array}{l}\mathrm{BMl} \\
\left(\mathrm{kg} / \mathrm{m}^{2}\right)\end{array}$ & $\begin{array}{l}\text { SMl } \\
\left(\mathrm{kg} / \mathrm{m}^{2}\right)\end{array}$ & $\begin{array}{l}\text { Job } \\
(\mathrm{Pri} ; \mathrm{Sec} ; \mathrm{Ter} ; \mathrm{None})\end{array}$ \\
\hline Male & $50-59$ & 50 & $171.8(5.9)$ & $67.1(9.0)$ & $22.7(2.9)$ & $7.6(0.7)$ & $3 ; 7 ; 40 ; 0$ \\
& $60-69$ & 53 & $166.7(4.7)$ & $66.9(7.7)$ & $24.1(2.7)$ & $7.5(0.6)$ & $18 ; 5 ; 19 ; 11$ \\
& $70-79$ & 55 & $163.2(4.9)$ & $60.0(10.2)$ & $22.5(3.4)$ & $7.0(0.7)$ & $22 ; 2 ; 8 ; 23$ \\
& $80-89$ & 45 & $160.1(5.6)$ & $57.5(8.4)$ & $22.4(2.7)$ & $7.0(0.9)$ & $19 ; 0 ; 3 ; 23$ \\
& Total & 203 & $165.6(6.8)$ & $63.0(9.8)$ & $22.9(3.0)$ & $7.3(0.8)$ & $62 ; 14 ; 70 ; 57$ \\
& $50-59$ & 47 & $158.1(4.9)$ & $55.4(8.9)$ & $22.2(3.8)$ & $5.9(0.6)$ & $5 ; 4 ; 29 ; 9$ \\
& $60-69$ & 61 & $152.8(5.3)$ & $52.2(7.6)$ & $22.3(2.7)$ & $5.9(0.5)$ & $21 ; 4 ; 17 ; 19$ \\
& $70-79$ & 54 & $149.7(5.3)$ & $50.6(7.9)$ & $22.6(3.2)$ & $6.1(0.6)$ & $16 ; 4 ; 8 ; 26$ \\
& $80-89$ & 48 & $144.6(5.8)$ & $48.3(7.8)$ & $23.1(3.3)$ & $6.1(0.7)$ & $11 ; 0 ; 5 ; 32$ \\
\end{tabular}

Note: Values represent mean (standard deviation).

Abbreviations: BMI Body Mass Index; SMI Skeletal Muscle Index; Pri Primary industry employment; Sec Secondary industry employment; Ter Tertiary industry employment

45 for $80-89$ years. The number of female participants were 47 for those aged 50-59 years, 61 for 60-69 years, 54 for $70-79$ years, and 48 for $80-89$ years. There were many tertiary industry workers up to retirement at age 65 and agricultural workers in the post-retirement age group. The older people were, the more likely they were classified to be unemployed.

The physical functions of participants are shown for each age group and sex in Table 2 . All physical performance tests revealed inferior results on average for higher age groups. These physical characteristics of each group divided by age and sex demonstrated approximately the same value in 2014 for the Japanese population [23]. Three hundred and twelve patients $(75.5 \%)$ were diagnosed as LoS, of which 144 (46.2\%) were male and 168 (53.8\%) were female. The prevalence of LoS was $53.6 \%$ for patients aged 50-59 years, $60.5 \%$ for 60-69 years, $90.8 \%$ for $70-79$ years, and $98.9 \%$ for $80-89$ years. The severity of LoS for the 312 patients were 210 (67.3\%) for LoS Stage 1 and 102 (32.7\%) for LoS Stage 2. More than half of the participants aged 50-59, 60-69, and 70-79 were LoS Stage 1 . In both males and females, there was a significantly higher prevalence of LoS in older age groups, e.g., those over 60 years of age.

Over $60 \%$ of both male and female participants aged 80-89 years were LoS Stage 2. The prevalence of LoS and LoS Stage 2 significantly increased with age (Fig. 2). Due to difficulties in maintaining the one-leg stand from a 40-cm-high seat during the SUT, many participants were diagnosed as LoS under the LoS risk test (Table 3).

In comparing the knee extension strength and OLS test between the no LoS and LoS groups, the knee extension strength was significantly lower in the LoS group in males and females aged 50-59 years and females aged 60-69 years (Fig. 3). OLS test was significantly lower in value for males aged 60-69 in the LoS group (Fig. 4).
We were unable to compare participants aged 70-79 and 80-89 because participants were mostly classified under the LoS group.

The total number of participants diagnosed as sarcopenia was 31 (9.81\%), 23 of which were male $(11.3 \%)$ and 8 of which were female $(4.91 \%)$. The prevalence of sarcopenia also increased with age in males, consisting of $0 \%$ for participants aged 60-69 years, $12.7 \%$ for participants aged $70-79$, and $35.6 \%$ for participants aged $80-$ 89. In male participants, the prevalence of sarcopenia was significantly higher as the age strata grew higher (Fig. 5).

Forty-nine patients diagnosed with sarcopenia included 29 (93.5\%) with LoS, 11 (35.5\%) classified as LoS stage 1 , and $18(58.1 \%)$ classified as stage 2 . Participants with sarcopenia tended to have LoS, but the association was not significant (odds ratio [OR] 3.4, 95\% confidence interval [95\% CI] 0.8 to $14.6, P=0.10)$. The OR for males and females were 5.5 (95\%CI 0.7 to $42.7, P=0.10)$ and $1.5(95 \% \mathrm{CI} 0.2$ to $13.1, P=0.69)$, respectively. There were no significant associations for both sex. Table 4 shows whether the presence of sarcopenia can predict the presence of LoS. Although the screening for LoS via diagnosis of sarcopenia was insensitive and often overlooked, the specificity was higher at $95 \%$ or more. The positive/negative likelihood ratio were $3.12 / 0.92$, respectively, which were insufficient for predicting LoS based on the diagnosis of sarcopenia.

\section{Discussion}

In the present study, we evaluated the prevalence of LoS and sarcopenia. Furthermore, we assessed the knee extension strength, balancing ability, and sarcopenia in relation to LoS. The total number of participants was 312 (75.5\%) for those diagnosed as LoS and 31 for those diagnosed as sarcopenia (9.81\%). Knee extension strength 
Table 2 Results of physical performance tests in each subject group

\begin{tabular}{llllllll}
\hline & Age strata (years) & Knee extension strength & OLS time (sec) & Grip strength $(\mathrm{kg})$ & SUT & TST score & 25-qGLFS score \\
\hline Male & $50-59$ & $1.8(0.5)$ & $51.5(13.4)$ & $40.0(6.9)$ & 5 & $1.6(0.2)$ & $2.9(3.5)$ \\
& $60-69$ & $1.7(0.5)$ & $46.0(16.9)$ & $36.8(6.0)$ & 4 & $1.5(0.2)$ & $5.1(5.6)$ \\
& $70-79$ & $1.3(0.4)$ & $24.6(17.1)$ & $31.1(5.2)$ & 4 & $1.4(0.2)$ & $9.4(11.1)$ \\
& $80-89$ & $1.0(0.4)$ & $13.2(13.7)$ & $25.1(5.2)$ & 3 & $1.1(0.3)$ & $14.3(14.1)$ \\
Female & $50-59$ & $1.4(0.4)$ & $46.9(16.2)$ & $25.4(5.2)$ & 4 & $1.5(0.1)$ & $5.0(4.8)$ \\
& $60-69$ & $1.2(0.4)$ & $43.2(17.0)$ & $21.8(3.9)$ & 4 & $1.4(0.1)$ & $4.4(4.6)$ \\
& $70-79$ & $0.9(0.3)$ & $23.0(15.9)$ & $20.5(3.9)$ & 3 & $1.3(0.4)$ & $13.9(15.8)$ \\
& $80-89$ & $0.8(0.3)$ & $10.9(12.4)$ & $17.1(3.6)$ & 3 & $1.1(0.3)$ & $18.9(14.8)$ \\
\hline
\end{tabular}

Note: Values represent mean (standard deviation).

Abbreviations: OLS One-leg standing; SUT Stand-up test; TST Two-step test; 25-qGLFS 25-Question geriatric locomotive function scale

and OLS test between the no LoS and LoS groups, knee extension strength was significantly lower in the LoS group. Particularly in males, sarcopenia patients could be assumed to have LoS, but non-sarcopenia patients may or may not have LoS.

A characteristic feature of this study was the collection of participants with age ranging from 50 to 89 years by randomly sampling from the resident register. Compared to conventional research on residents that recruits active volunteers, this research was designed to create a cohort that more accurately reflects common residents. In addition, another unique feature of this study was that we gathered approximately 50 participants for physical examinations in each age group and sex, and as a result, the age and male-female ratio of participants aged 5089 years were uniformly distributed. This uniform distribution is advantageous for performing accurate statistical comparisons between sexes or age groups.

The prevalence of LoS in this study increased with age, with approximately half of the participants aged 50 59 years and over $90 \%$ aged $70-79$ years and $80-89$ years who were diagnosed as LoS. There were approximately $43,376,300$ patients with $\operatorname{LoS}$ ( $75.5 \%$ of $50-89$ years), 29 , 195,593 patients $(50.8 \%)$ with LoS Stage 1, and 14,180, 716 patients $(24.7 \%)$ with LoS Stage 2 in Japan when weighted by the Japanese population. In previous studies [5-8] and in this study, there are differences in the composition ratio for age and sampling method of subjects; thus, cannot simply compare the rate of prevalence for LoS in this study with results from previous studies. However, the prevalence of LoS in this study was comparable to the prevalence of LoS described in a study by

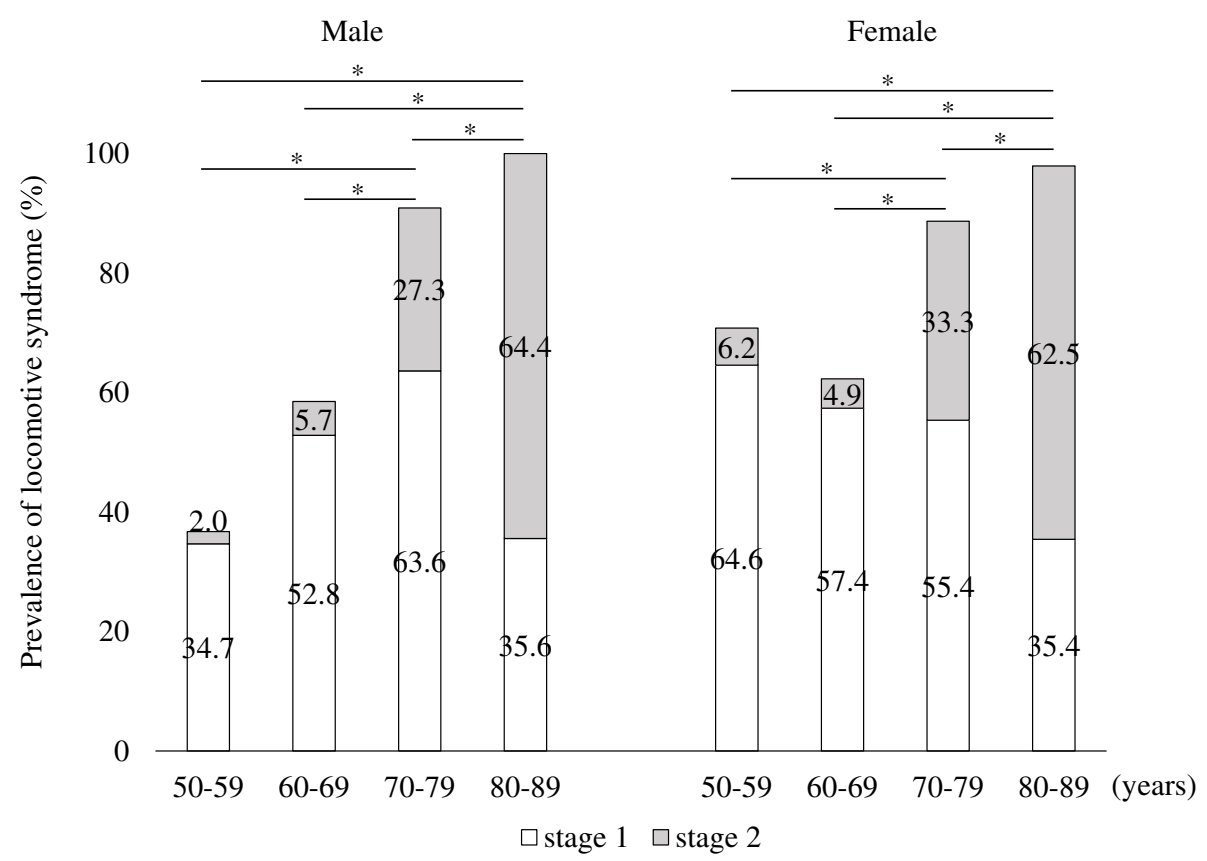

Fig. 2 Prevalence of locomotive syndrome. ${ }^{*} p<0.05$ 
Table 3 Prevalence by age and sex as evaluated in the locomotive syndrome risk test

\begin{tabular}{llllll}
\hline & Age strata (years) & $N$ & Unable to complete one-leg SUT from $\geq 40 \mathrm{~cm}$ & TST $<1.3$ & $25-\mathrm{qGLFS}$ score $\geq 7$ \\
\hline Male & $50-59$ & 50 & $14(28.0 \%)$ & $2(4.0 \%)$ & $6(12.0 \%)$ \\
& $60-69$ & 53 & $28(52.8 \%)$ & $5(9.4 \%)$ & $12(22.6 \%)$ \\
& $70-79$ & 55 & $49(89.1 \%)$ & $16(29.1 \%)$ & $22(40.0 \%)$ \\
Female & $80-89$ & 45 & $45(100 \%)$ & $32(71.1 \%)$ & $28(62.2 \%)$ \\
& $50-59$ & 47 & $26(55.3 \%)$ & $1(2.1 \%)$ & $13(27.7 \%)$ \\
& $60-69$ & 61 & $32(52.5 \%)$ & $8(13.1 \%)$ & $12(19.7 \%)$ \\
& $70-79$ & 54 & $48(88.9 \%)$ & $20(37.0 \%)$ & $30(55.6 \%)$ \\
& $80-89$ & 48 & $46(95.8 \%)$ & $37(77.1 \%)$ & $41(85.4 \%)$ \\
\hline
\end{tabular}

Note: Values represent number (prevalence)

Abbreviations: SUT Stand-up test; TST Two-step test; 25-qGLFS 25-Question geriatric locomotive function scale

Yoshimura et al. [5] that calculated the rate of prevalence by age and sex. On the other hand, when comparing the ratio of participants who were diagnosed with LoS by each criterion in the LoS risk test, the ratio in this study was greater than in the study by Yoshimura et al. in terms of those with difficulties in maintaining a one-leg stand from a $40-\mathrm{cm}$-high seat during the SUT. In contrast, the ratio of participants who obtained a TST score $>1.3$ was less than what was reported by Yoshimura et al. There was no significant difference between both studies in terms of the results in 25-qGLFS. The reason for the difference in results for each criterion in diagnosing LoS is unknown. However, there are reports that suggest that the average step count increases with size of the city [24] and that those who exercise at a moderate or higher intensity are greater in urban areas than in rural areas [25]. We suspect that the results of the SUT in this study were inferior to other studies due to decrease in the muscle strength of knee extension as a result of the comparatively small amount of daily physical activity in residents of rural areas.

When comparing the physical function between the no LoS and LoS groups, the knee extension strength was significantly lower in both male and female participants with $\mathrm{LoS}$ aged $50-59$ years, and female participants aged 60-69 years. From this fact, we believe that some measures to prevent or improve LoS may require exercise to increase the muscle strength of the lower limbs.

In terms of the prevalence of sarcopenia in the general elderly population of Japan, one report described that the prevalence of sarcopenia according to age group stratifications of $60-64,65-69,70-74,75-79$, and $\geq 80$ years were $0.5,0.0,4.3,11.2$, and $27.0 \%$, respectively [26]. The prevalence of sarcopenia was higher when

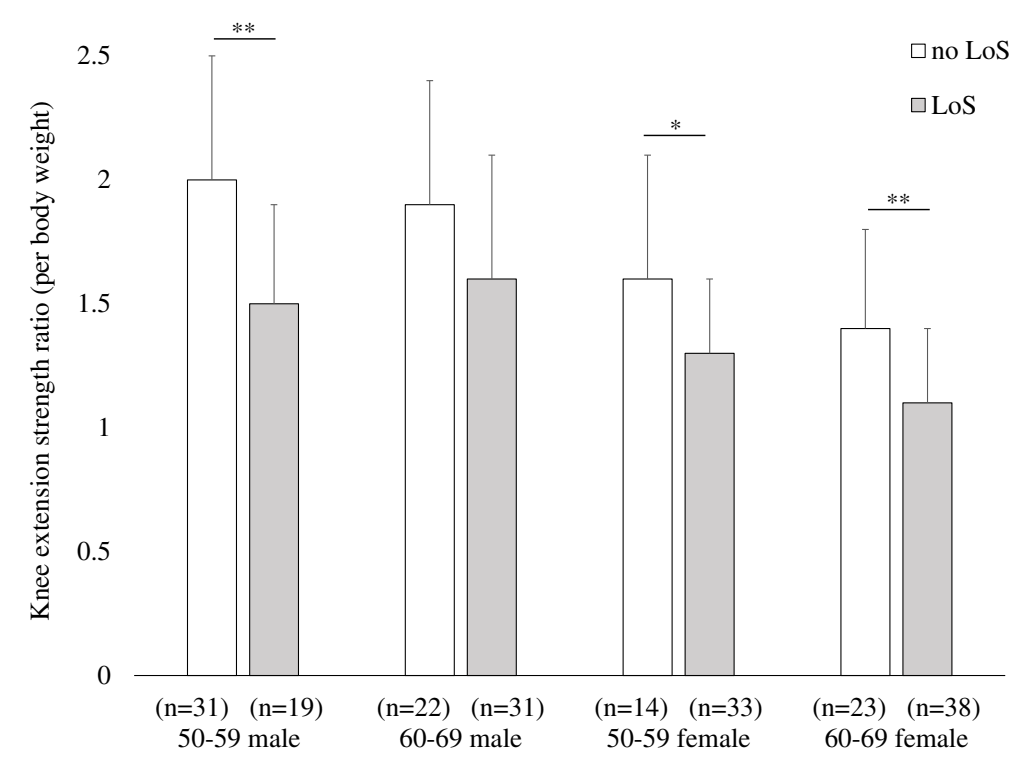

Fig. 3 Comparison of knee extension strength between participants indicated no-locomotive syndrome and those indicated locomotive syndrome. Values represent mean (standard deviation). LoS, locomotive syndrome. ${ }^{*} p<0.05 ;{ }^{* *} p<0.01$ 


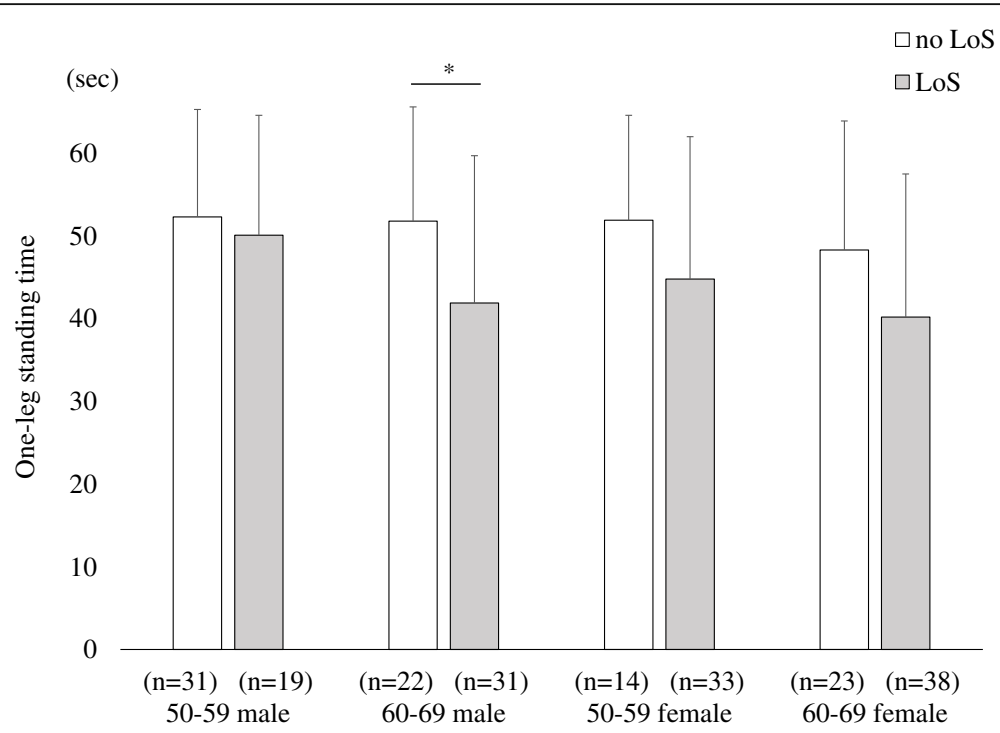

Fig. 4 Comparison of one-leg standing test between participants indicated no-locomotive syndrome and those indicated locomotive syndrome. Values represent mean (standard deviation). LoS, locomotive syndrome. ${ }^{*} p<0.05$

comparing each age group with past reports. In the present study, the total prevalence of sarcopenia was 9.81\%, which was slightly higher than in previous studies. There were approximately 4,031,275 patients with sarcopenia $(7.02 \%$ of $60-89$ years $)$ in Japan when weighted by the Japanese population.

While there have been reports that describe no sex difference in the prevalence of $\operatorname{LoS}$ and sarcopenia $[6,26$, 27], there have also been reports that describe a higher prevalence of sarcopenia in males compared to females [28]. Significant associations between sarcopenia and
LoS were found in both sex based on the results of the ROAD study, which is a study of Japanese residents aged 60 and over [15]. On the other hand, the results of this study showed that males might have an association between sarcopenia and LoS, while females might not. There are several possibilities as to why this difference may have occurred. First, the sample size may have been insufficient. The results for females could be clarified with a larger sample population. However, other causes should also be considered. The cause of LoS, which is more common in females, might have been implicated.

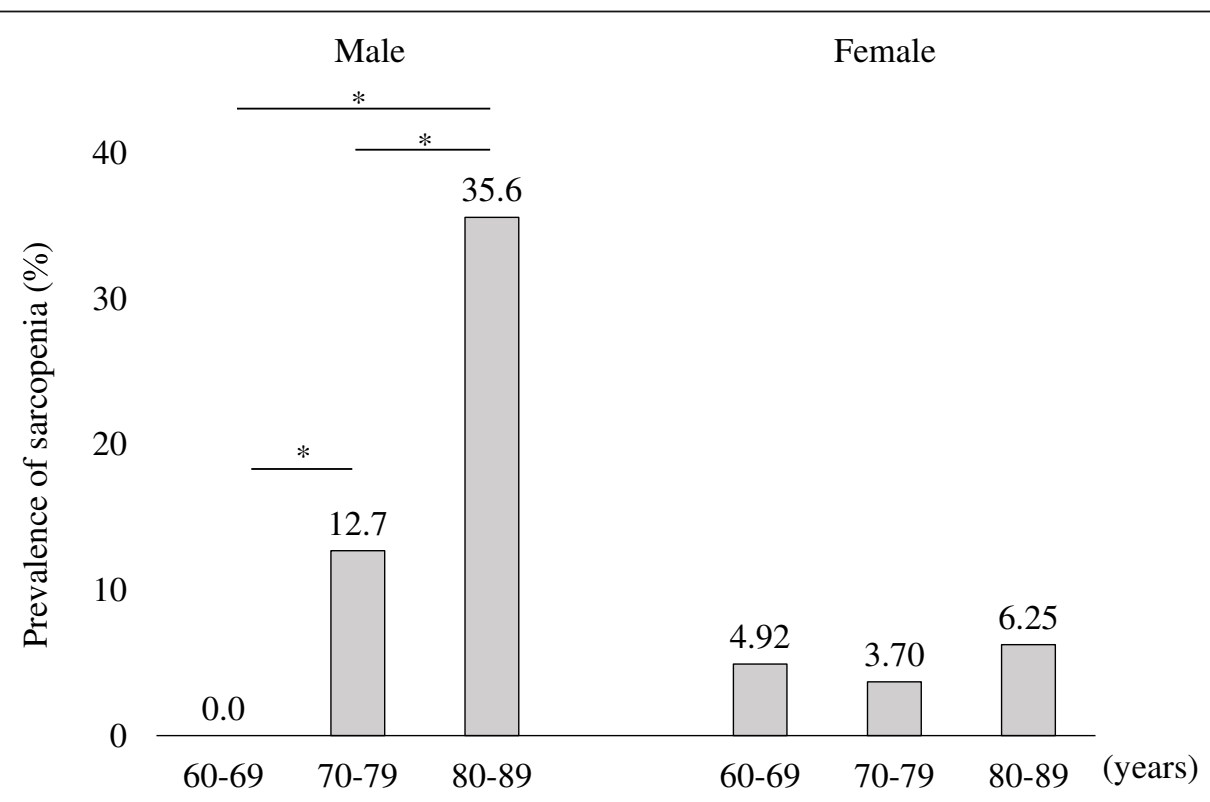

Fig. 5 Prevalence of sarcopenia. ${ }^{*} p<0.05$ 
Table 4 Screening for locomotive syndrome based on the diagnosis of sarcopenia

\begin{tabular}{lllll}
\hline & Sensitivity & Specificity & Positive likelihood ratio & Negative likelihood ratio \\
\hline Male & $17 \%(15-18 \%)$ & $96 \%(83-99 \%)$ & $4.71(0.91-27.4)$ & $0.86(0.82-1.01)$ \\
Female & $5 \%(3-6 \%)$ & $97 \%(87-99 \%)$ & $1.51(0.26-9.39)$ & $0.98(0.95-1.11)$ \\
Total & $11 \%(10-12 \%)$ & $96 \%(89-99 \%)$ & $3.12(0.88-11.8)$ & $0.92(0.89-1.01)$ \\
\hline
\end{tabular}

Note: Values represent with (95\% confidence intervals)

For example, osteoporosis and knee osteoarthritis are common in females [6]. Walking speed, which could not be assessed in our study, may contribute to a more severe diagnosis of sarcopenia for females than for males. Moreover, sex differences of the muscle mass cut-off in the diagnostic criteria for sarcopenia might have been affected.

There are several limitations in this study. First, although the research design reduces the sampling bias by adopting random sampling from the resident register, we may not have been able to control for all potential biases as a result of the low participation rate. Furthermore, this study may not reflect the regional characteristics of urban residents, because it is a sampling of one town. Subjects were randomly sampled from the resident registry of the town, but the decision to participate in this study was left to the sampled subjects. Therefore, we conducted a sensitivity analysis on the association between sarcopenia and LoS, assuming that there was heterogeneity in the participation rate depending on whether or not the participants felt impaired in terms of their mobility. While the overall participation rate $(32 \%)$ remained constant, we changed the participation rates of each cluster, which were LoS $(+)$ / Sarcopenia (+), LoS (+) / Sarcopenia (-), LoS(-) / Sarcopenia(+), and $\operatorname{LoS}(-) /$ Sarcopenia(-), to $20,30,40$, and $50 \%$. These participation rates resulted in an adjusted odds ratio of 4.1 between sarcopenia and LoS, which was larger than the crude odds ratio of 3.4. Conversely, when we changed the participation rates of the abovementioned clusters to $50,40,30$, and $20 \%$, the adjusted odds ratio was 4.1 , which was also larger than the crude odds ratio of 3.4. Assuming the impact of heterogeneity in participation rates due to the small sample size, it is suggested that we were likely underestimating the relationship between sarcopenia and LoS. Secondly, the diagnosis of sarcopenia is underestimated because we could not measure walking speed in this survey. This has a severe impact on the results of analysis for association between sarcopenia and LoS. Therefore, we performed a sensitivity analysis for sarcopenia misclassification as an additional analysis, and the results are presented in Table 5. The crude odds ratio for sarcopenia/LoS was 3.4 in this study. Because participants with LoS are expected to have low walking speed, the false-negative rate of sarcopenia for LoS-positive participants is likely to be higher than that of LoS-negative participants. Accordingly, the adjusted odds ratio would be greater. Sarcopenia may prove to be a predictor of LoS with the introduction of an appropriate diagnostic procedure for sarcopenia.

\section{Conclusion}

A characteristic feature of this study was the collection of participants with age ranging from 50 to 89 years by randomly sampling from the resident register. Therefore, this research was designed to create a cohort that more accurately reflects common residents. The prevalence of LoS in this study increased with age, with approximately half of the participants aged $50-59$ years and over $90 \%$ aged 70 79 years and $80-89$ years who were diagnosed as LoS. Due to difficulties in maintaining the one-leg stand from a 40$\mathrm{cm}$-high seat during the SUT, many participants were diagnosed as LoS. Moreover, the knee extension strength was significantly lower in both male and female participants with LoS aged 50-59 years, and female participants aged 60-69 years. From this fact, we believe that some measures to prevent or improve LoS may require exercise to increase the muscle strength of the lower limbs.

Table 5 Sensitivity analysis for misclassifications of sarcopenia

\begin{tabular}{|c|c|c|c|c|c|c|}
\hline \multirow[t]{2}{*}{ Sensitivity of sarcopenia diagnosis in LoS (+) } & \multicolumn{6}{|c|}{ Sensitivity of sarcopenia diagnosis in LoS (-) } \\
\hline & $100 \%$ & $90 \%$ & $80 \%$ & $70 \%$ & $60 \%$ & $50 \%$ \\
\hline $100 \%$ & 3.4 & 3.0 & 2.7 & 2.3 & 2.0 & 1.6 \\
\hline $90 \%$ & 3.8 & 3.4 & 3.0 & 2.6 & 2.2 & 1.8 \\
\hline $80 \%$ & 4.4 & 3.9 & 3.5 & 3.0 & 2.6 & 2.1 \\
\hline $70 \%$ & 5.1 & 4.6 & 4.1 & 3.5 & 3.0 & 2.5 \\
\hline $60 \%$ & 6.2 & 5.5 & 4.9 & 4.3 & 3.6 & 3.0 \\
\hline $50 \%$ & 7.8 & 7.0 & 6.1 & 5.3 & 4.5 & 3.7 \\
\hline
\end{tabular}

Note: Values represent adjusted odds ratio between sarcopenia and locomotive syndrome 


\section{Abbreviations}

LoS: Locomotive syndrome; 25-qGLFS: 25-Question geriatric locomotive function scale; SUT: Stand-up test; TST: Two-step test; OLS: One-leg standing; SMI: Skeletal muscle mass index; BMI: Body mass index; Pri: Primary industry employment; Sec: Secondary industry employment; Ter: Tertiary industry employment; OR: Odds ratio; 95\% Cl: 95\% Confidence interval

\section{Acknowledgements}

We would like to thank Dr. Takashi Igarashi of the Center for Clinical Research at Shinshu University Hospital for date management. We would also like to thank Dr. Hironobu Sato of the Obuse Town Institute for Community Health Promotion and the Obuse town office for sample selection in this study.

\section{Authors' contributions}

RT: study conception and design, participant assessment, data collection, statistical analysis, and writing manuscript. Sl: study conception and design, statistical analysis, and writing manuscript. Yl: study conception and design, participant assessment, and data collection. AS: participant assessment, and data collection. NS: study conception and design, participant assessment, and data collection. JT: initiated the study, have drafted the work, and supervised all aspects of the work. HH: initiated the study, have drafted the work, and supervised all aspects of the work. HK: initiated the study, have drafted the work, critical review of manuscript, and supervised all aspects of the work. All authors have read and approved the final manuscript.

\section{Funding}

This work was supported by a grant from the Japan Orthopaedics and Traumatology Research Foundation, Inc. (no. 339), Project Research Funds from the Japanese Orthopaedic Association, the Promotion Project of Education, Research, and Medical Care from Shinshu University Hospital, Research Funding from the Japanese Society for Musculoskeletal Medicine, and The Nakatomi Foundation. All funds were used to travel expenses, reward for experiment staff, office supplies in this research, data maintenance, and grammar proofreading of manuscripts. They were not used in study design, data interpretation, or analysis.

\section{Availability of data and materials}

The datasets used and analysed during the current study are available from the corresponding author on reasonable request.

\section{Ethics approval and consent to participate}

The protocols in this study conformed to the ethical guidelines of the 2013 Declaration of Helsinki. Informed consent was obtained from participants prior to the initiation of study. Participants were informed about the purposes of the research both verbally and in writing prior to the study, and written informed consent was obtained from all participants. This study was approved by the institutional review board of Shinshu University Hospital (approval number: 2792)

\section{Consent for publication}

Not applicable.

\section{Competing interests}

The authors declare that they have no competing interests.

\section{Author details}

${ }^{1}$ Rehabilitation Center, Shinshu University Hospital, Asahi 3-1-1, Matsumoto, Nagano 390-8621, Japan. ²Department of Orthopaedic Surgery, Shinshu University School of Medicine, Asahi 3-1-1, Matsumoto, Nagano 390-8621, Japan. ${ }^{3}$ Department of Orthopaedic Surgery, New Life Hospital, Obuse, Kamitakai-gun, Nagano 381-0295, Japan.
\end{abstract}

Received: 17 March 2020 Accepted: 29 June 2020

Published online: 03 July 2020

\section{References}

1. Nakamura K. A "super-aged" society and the "locomotive syndrome." J Orthop Sci 2008;13(1):1-2.

2. Ishibashi H. Locomotive syndrome in Japan. Osteoporos Sarcopenia. 2018; 4(3):86-94.
3. Seichi A, Hoshino Y, Doi T, Akai M, Tobimatsu Y, Iwaya T. Development of a screening tool for risk of locomotive syndrome in the elderly: the 25question geriatric locomotive function scale. J Orthop Sci. 2012;17(2):163-72.

4. Nakamura K, Ogata T. Locomotive syndrome: definition and management. Clin Rev Bone Miner Metab. 2016;14(2):56-67.

5. Yoshimura N, Muraki S, Oka H, Tanaka S, Ogata T, Kawaguchi H, Akune T, Nakamura K. Association between new indices in the locomotive syndrome risk test and decline in mobility: third survey of the ROAD study. J Orthop Sci. 2015;20(5):896-905.

6. Yoshimura N, Muraki S, Nakamura K, Tanaka S. Epidemiology of the locomotive syndrome: the research on osteoarthritis/osteoporosis against disability study 2005-2015. Mod Rheumatol. 2017;27(1):1-7.

7. Nakatoh S. Usefulness of conducting locomotive syndrome screening along with specific health checkups for spreading the awareness of this screening Rinsho Seikeigeka (Clin Orthop Surg). 2016;51(1):19-22 (in Japanese).

8. Imagama S, Hasegawa Y, Ando K, Kobayashi K, Hida T, Ito K, Tsushima M, Nishida $Y$, Ishiguro N. Staged decrease of physical ability on the locomotive syndrome risk test is related to neuropathic pain, nociceptive pain, shoulder complaints, and quality of life in middle-aged and elderly people - the utility of the locomotive syndrome risk test. Mod Rheumatol. 2017;27(6): 1051-6.

9. Tanaka S, Ando K, Kobayashi K, Hida T, Seki T, Hamada T, Ito K, Tsushima M, Morozumi M, Machino M, Ota K, Ishiguro N, Hasegawa Y, Imagama S. Increasing postural sway in balance test is related to locomotive syndrome risk: a cross-sectional study. J Orthop Sci. 2019;24(5):912-7.

10. Imagama S, Hasegawa Y, Ando K, Kobayashi K, Hida T, Ito K, Tsushima M, Nishida $Y$, Ishiguro N. Staged decrease of physical ability on the locomotive syndrome risk test is related to neuropathic pain, nociceptive pain, shoulder complaints, and quality of life in middle-aged and elderly people - the utility of the locomotive syndrome risk test. Mod Rheumatol. 2017;27(6): 1051-6.

11. Kasukawa $Y$, Miyakoshi N, Hongo M, Ishikawa $Y$, Kudo D, Kijima H, Kimura R, Ono Y, Takahashi Y, Shimada Y. Lumbar spinal stenosis associated with progression of locomotive syndrome and lower extremity muscle weakness. Clin Interv Aging. 2019;14:1399-405.

12. Chen LK, Liu LK, Woo J, Assantachai P, Auyeung TW, Bahyah KS, Chou MY, Chen LY, Hsu PS, Krairit O, Lee JS, Lee WJ, Lee Y, Liang CK, Limpawattana P, Lin CS, Peng LN, Satake S, Suzuki T, Won CW, Wu CH, Wu SN, Zhang T, Zeng P, Akishita M, Arai H. Sarcopenia in Asia: consensus report of the Asian working Group for Sarcopenia. J Am Med Dir Assoc. 2014;15(2):95-101.

13. Landi F, Liperoti R, Fusco D, Mastropaolo S, Quattrociocchi D, Proia A, Russo A, Bernabei R, Onder G. Prevalence and risk factors of sarcopenia among nursing home older residents. J Gerontol A Biol Sci Med Sci. 2012;67(1):48-55.

14. Ikemoto T, Arai YC. Locomotive syndrome: clinical perspectives. Clin Interv Aging. 2018;30(13):819-27.

15. Yoshimura N, Muraki S, lidaka T, Oka H, Horii C, Kawaguchi H, Akune T, Nakamura K, Tanaka S. Prevalence and co-existence of locomotive syndrome, sarcopenia, and frailty: the third survey of research on osteoarthritis/osteoporosis against disability (ROAD) study. J Bone Miner Metab. 2019;37(6):1058-66.

16. Muranaga S. Evaluation of the muscular strength of the lower extremities using the standing movement and clinical application. Showa Ikaishi (J Showa Med Assoc). 2001;61(3):362-7 (in Japanese).

17. Muranaga S, Hirano K. Development of a convenient way to predict ability to walk, using a two-step test. Showa Ikaishi (J Showa Med Assoc). 2003; 63(3):301-8 (in Japanese).

18. Neil SE, Myring A, Peeters MJ, Pirie I, Jacobs R, Hunt MA, Garland SJ, Campbell KL. Reliability and validity of the performance recorder 1 for measuring isometric knee flexor and extensor strength. Physiother Theory Pract. 2013;29(8):639-47.

19. Flansbjer UB, Lexell J. Reliability of knee extensor and flexor muscle strength measurements in persons with late effects of polio. J Rehabil Med. 2010; 42(6):588-92.

20. Lee JW, Kang JS, Park SJ, Yoon SW, Jeong SK, Heo M. Effects of interelectrode distance on delayed onset muscle soreness in microcurrent therapy. J Phys Ther Sci. 2013;25(11):1451-4.

21. Ministry of Education, Culture, Sports, Science and Technology, Guidelines for implementing a new physical examination (subjects aged 65-79) with an open-eyes one-leg standing test (Internet). 2010 (cited 2018 Dec). Available from: http://www.mext.go.jp/component/a_menu/sports/detail/_ icsFiles/afieldfile/2010/07/30/1295079_04.pdf (in Japanese). 
22. Kanda Y. Investigation of the freely available easy-to-use software 'EZR' for medical statistics. Bone Marrow Transplant. 2013;48(3):452-8.

23. Ministry of Health, Labour and Welfare. Part 2 Health and hygiene Chapter 1 Health Tables 2-6 Average height/weight and sex by year and age (Internet). 2017 (cited 2018 Mar). Available from: https://www.mhlw.go.jp/ toukei/youran/indexyk_2_1.html (in Japanese).

24. Ihara M, Inoue S, Takamiya T, Ohya Y, Odagiri Y, Fukushima N, Hayashi T, Kikuchi $\mathrm{H}$, Sato H, Shimomitsu T. A cross-sectional study of the association between city scale and daily steps in Japan: data from the National Health and nutrition survey Japan (NHNS-J) 2006-2010. Nihon Koshu Eisei Zasshi (Japanese J Public Health). 2016;63(9):549-59 (in Japanese).

25. Fan JX, Wen M, Kowaleski-Jones L. Rural-urban differences in objective and subjective measures of physical activity: findings from the National Health and nutrition examination survey (NHANES) 2003-2006. Prev Chronic Dis. 2014:11:E141.

26. Yoshimura N, Muraki S, Oka H, lidaka T, Kodama R, Kawaguchi H, Nakamura K, Tanaka S, Akune T. Is osteoporosis a predictor for future sarcopenia or vice versa? Four-year observations between the second and third ROAD study surveys. Osteoporos Int. 2017;28(1):189-99.

27. Tanishima S, Hagino H, Matsumoto H, Tanimura C, Nagashima H. Association between sarcopenia and low back pain in local residents prospective cohort study from the GAINA study. BMC Musculoskelet Disord. 2017;18(1):452

28. Trajanoska K, Schoufour JD, Darweesh SK, Benz E, Medina-Gomez C, Alferink L, Lahousse L, Brusselle G, Stricker B, Darwish Murad S, Zillikens MC, Uitterlinden AG, Ikram MA, Franco OH, Rivadeneira F. Sarcopenia and its clinical correlates in the general population: the Rotterdam study. J Bone Miner Res. 2018;33(7):1209-18.

\section{Publisher's Note}

Springer Nature remains neutral with regard to jurisdictional claims in published maps and institutional affiliations.

Ready to submit your research? Choose BMC and benefit from:

- fast, convenient online submission

- thorough peer review by experienced researchers in your field

- rapid publication on acceptance

- support for research data, including large and complex data types

- gold Open Access which fosters wider collaboration and increased citations

- maximum visibility for your research: over $100 \mathrm{M}$ website views per year

At $\mathrm{BMC}$, research is always in progress.

Learn more biomedcentral.com/submissions 